\section{Field-of-view tests for a virtual cockpit application}

\author{
M. Chevaldonné, ${ }^{\text {a,b }}$ C. Ballaz, ${ }^{\mathrm{c}}$ F. Mérienne, \\ M. Neveu, ${ }^{a}$ N. Chevassus, ${ }^{b}$ F. Guillaume, ${ }^{b}$ and \\ C. Arbez ${ }^{a}$ \\ ${ }^{\mathrm{a}}$ Le2i (UMR CNRS 5158), Institut Image_ENSAM \\ 2, rue Thomas Dumorey \\ 71321 Chalon sur Saône-France \\ E-mail: marc.chevaldonne@cluny.ensam.fr. \\ ${ }^{\mathrm{b}} \mathrm{EADS}-\mathrm{CCR}$ \\ 12, rue Pasteur \\ 92152 Suresnes Cedex-France \\ ${ }^{\mathrm{c}}$ Laboratoire de Psychologie Expérimentale \\ Université de Genève \\ 40 bv. Du pont d'Arve \\ 1205 Genève-Suisse
}

\begin{abstract}
The virtual cockpit application aims to perform ergonomics studies on future aircraft cockpits, directly from digital mock-ups. This is made possible by immersing a user in a digital mock-up with the help of virtual reality devices. The challenge is then to reproduce a realistic and exact visual environment. To do that, some visual criteria have to be determined: the necessary values of visual acuity, temporal resolution, and field of view. Some of them are easy to find, but the field-of-view requirement is far more subjective and complex to evaluate. We present tests which have helped us to determine the necessary and sufficient values of field of view for such an application. We advise then to use a field of view higher than $75 \mathrm{deg}$, for ergonomic studies and good performances of the users, and up to 133 deg to increase visual comfort. These results will be used for the specification of a head-mounted display for the application. (C) 2006 SPIE and IS\&T. [DOI: 10.1117/1.2189211]
\end{abstract}

\section{Introduction}

For years, the production process has tended to decrease the time to market in the aircraft industry. Concurrent engineering gives powerful opportunities for conception and production of aircraft cockpits. It is largely based on digital mock-ups (DMU), as they enable communication between numerous professionals working on the project. A DMU is a numerical version of a product containing all the information relative to it. It is easy to upgrade and to adapt. This enables to follow a product all along its life cycle. A physical mock-up (PMU) is a physical version of a product, containing part of the information relative to it, at a given time, and then does not permit following the product during its life cycle. Design processes based on DMU allow savings of time and money by decreasing the number of PMU. However, it is difficult to take into account human factors within DMU, because human-product interactions are not well taken into consideration in DMU. That is why PMU are often used for human factors studies. A good alternative to PMU are virtual mock-ups (VMU). They are based on DMU and enable immersion and interaction within a virtual representation of the product. Thus, they allow us to evaluate concepts and detect issues linked to the ergonomics or

Paper 05151LRR received Sep. 2, 2005; revised manuscript received Feb. 3, 2006; accepted for publication Feb. 3, 2006; published online May 2, 2006.

$1017-9909 / 2006 / 15(2) / 020501 / 3 / \$ 22.00$ @ 2006 SPIE and IS\&T. other human factors. They also benefit from advantages similar to the DMU: fast preparation and simple upgrades.

The motive of our work is to give tools, software, and methods to allow the production of VMU.

The virtual cockpit application consists of the immersion of a user in a VMU, based on the DMU of an aircraft cockpit developed by cockpit designers, with the help of virtual reality devices. The user can then see the environment and interact with it.

In order to allow ergonomics studies, the application must give the user the opportunity to perceive and interact with the VMU with sufficient quality. Visual virtual perception is very dependent on several factors. Field of view (FOV) is one of them. The virtual cockpit application would benefit from the knowledge of the necessary FOV as it would help to choose an adapted head-mounted display (HMD) and calculate images of high quality.

This paper deals with the qualification of the field of view in the virtual cockpit application.

\section{Human Field of View in Virtual Environments or Cockpit Applications}

The human FOV represents the part of space visible by the eyes. It reaches $200 \mathrm{deg}$ horizontally and $135 \mathrm{deg}$ vertically. ${ }^{1}$ For one application, the whole FOV is not always necessary. Moreover, it will lead to complex optical developments. Our goal is then to define what is useful for the application, which will help to choose or specify an adapted visualization display that will not degrade perception.

Arthur ${ }^{1}$ has studied the influence of FOV on user performances during navigation or search tasks in virtual environments. He showed that there is an increase of performances when FOV increases and is lower than 100 deg. Related works in real environments with helicopter cockpits $^{2}$ or virtual environments but with noncockpit tasks ${ }^{3}$ prove that there is an increase of performances when FOV increases, until it reaches a limit value, depending on the task, between 40 and $80 \mathrm{deg}$. These studies allow us to suggest that a limit value around 80 deg horizontally might be sufficient. However, these results are not applicable to our application: the studies of Arthur ${ }^{1}$ have been conducted in virtual environments different for aircraft cockpits; the work of Kasper $e t a l{ }^{2}$ have been led on flying tasks in real helicopters; the tests of Schiefele ${ }^{3}$ were made in virtual cockpits, but for tasks different from visual ergonomics. Therefore, we have developed tests on different participants, in order to determine correctly the limit values to apply to our virtual cockpit application. These tests are based on user performances on specific tasks and on subjective self-assessment by the participants.

\section{Investigation of Limit Values of Field of View in the Virtual Cockpit Application}

This experiment has been separated into two tests. The first one has studied the influence of FOV on the user performance (warnings detection). The second one has studied the influence of FOV on participant visual comfort.

Our goal is to investigate the necessary and sufficient values of FOV, linked to the application, user visual comfort, and user performance.

Our hypothesis is as follows. Two limit values of FOV exist and are linked to the user and to the application: a 


\section{J E I L E T T E R S}

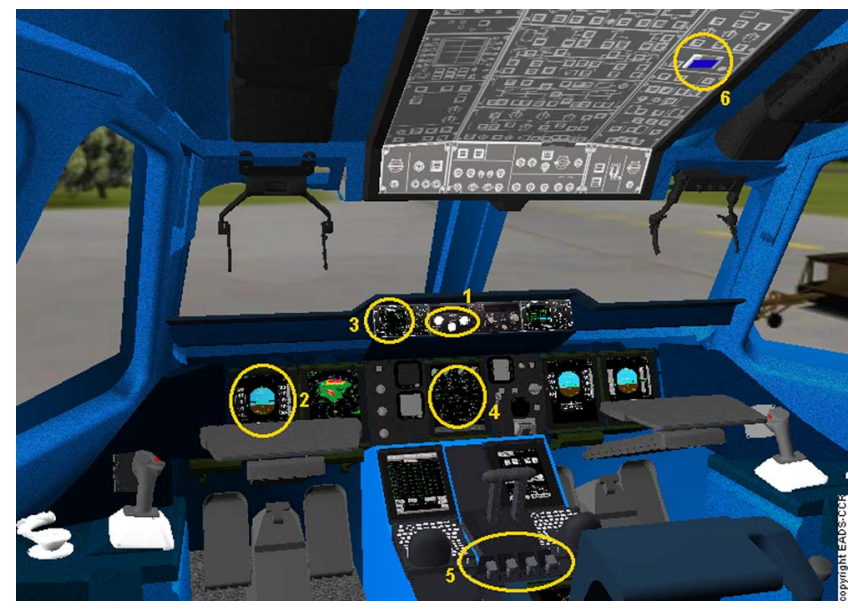

Fig. 1 The different elements of interaction during the tests.

minimum value below which performance and/or visual comfort are too degraded and a maximum value over which there is no increase in either performance or visual comfort for the user. Any FOV between these two values is sufficient to allow the application even if there may be an increase in performance and/or visual comfort between them.

Twenty-one persons participated in the test, but one of them successfully performed only $10 \%$ of the trial, thus only 20 data sets were analyzed. They were aged between 21 and 60 (mean 26). Four of them were familiar with cockpits, 10 with virtual reality, none with the application.

We used the following materials: (1) the MoVE of the Institut Image (a CAVE ${ }^{\text {TM}}$-like system), which allows us to use the VMU of the virtual cockpit, and FOV from 0 to $180 \mathrm{deg}$, by using a virtual window oriented in the direction of the head, and outside which the user sees only black; (2) special stereo glasses (made at the Institut Image) allowing a maximum FOV of 180 deg horizontally by 60 deg vertically; (3) a head tracking system in order to refresh images taking into account the head position and direction; and (4) a remote control, tracked in position and orientation and equipped with buttons and a virtual laser, in order to interact with buttons of the virtual cockpit.

During the test, the participants sat on the virtual pilot seat and were asked to interact with the cockpit by performing two tasks. The first task was to manipulate three buttons (see number 1 on Fig. 1) with the remote control to change three values on a flight display (number 2), in order to make them correspond with three asked values displayed on another flight screen (number 3). The second task was to detect visual warning signals on a flight display (number 4) by pressing a specific button of the remote control (the signal is then considered as detected), and to stop them with the remote control on two different possible places (numbers 5 and 6). Each signal lasts $10 \mathrm{~s}$ and always appears on the flight display (number 4) and randomly between these two places during the application.

These two tasks were advised by cockpit designers and test pilots, in order to immerse the user in the condition of use of the virtual cockpit application. The center of interest then is only the interaction between the participant and the cockpit interface. They require the participant to move the head or the eye, in order to verify or detect information.

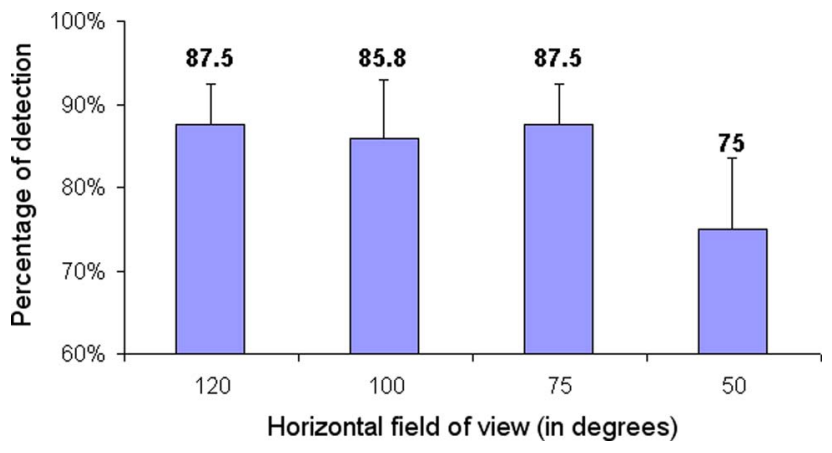

Fig. 2 Percentages and standard errors of warning detection by the participants as a function of the field of view..

\subsection{Test 1: Does FOV Have an Influence on User Performance (Warning Detection)}

Experimental plan and procedure. The test begins with a training phase during which the participant learns to perform the two tasks with a full FOV $(180 \mathrm{deg})$. After the training, test 1 begins. Vertical FOV is always $60 \mathrm{deg}$. Horizontal FOV changes randomly between the following values: $120,100,75$, and 50 deg. Each FOV lasts 1.5 min. All along the test, users have to perform the two tasks simultaneously, with a higher priority on the second task.

Collected data. For each FOV, the data collected are the number of detected and undetected warnings.

Results and discussion. Figure 2 shows the percentages of detection according to the FOV. For each FOV, we have divided the number of warnings detected by all the participants by the number of warnings sent to all of them. The detection percentage at the end of the training phase ( $180 \mathrm{deg}$ of FOV) is $90 \%$. Between 120 and $75 \mathrm{deg}$ there is no significant variation. A matrix of t-tests for dependant samples showed that only one paired comparison is significant. Participants were statically less efficient in detection at $50 \mathrm{deg}$ compared to $120 \mathrm{deg}[t(19)=2.52, \mathbf{p} \leqslant 0.02]$. No other paired comparisons were significant.

Our conclusion is that there is a risk of decrease in user performance in task 2, when FOV reaches values under $75 \mathrm{deg}$. This value is similar to the ones found by other research teams, ${ }^{1-3}$ that is, between 40 and $80 \mathrm{deg}$.

\subsection{Test 2: What Is the Influence of FOV on User Visual Comfort?}

This test aims to determine the limit values linked to visual comfort. The user had to determine the two values, but in a first phase, he had to change the FOV, and in a second phase, it changed automatically. The minimum value is defined as the FOV below which it is too annoying to work with over a span of several hours. The user should feel visually comfortable with any FOV higher than this value. Maximum value is defined as the FOV below which he perceives the first reduction of visual comfort or FOV degradation. 
Experimental plan and procedure. Vertical FOV is always 60 deg during this test. Horizontal FOV can take any value between 0 and 180 deg every half degree.

During the first phase, the user has to modify the FOV with the remote control four times: twice he has to increase the FOV from 0 deg; twice he has to decrease it from $180 \mathrm{deg}$. Each time, the user has to determine minimum and maximum value. Before validating any value, the user has to perform tasks 1 and 2 at least once.

During the second phase, the user has to perform tasks 1 and 2 simultaneously. FOV changes automatically from 0 to $180 \mathrm{deg}$ and from 180 to $0 \mathrm{deg}$ two times each, at the speed of $2.5 \mathrm{deg} / \mathrm{s}$. The user has to indicate by voice when he reaches maximum and minimum values every time.

After each phase, the supervisor asks the user if he thinks that he has found his maximum and minimum values. Possible answers are "yes" or "no."

Collected data. The data collected are the minimum and maximum values of each user at each phase, and the answers to the question.

Results and analysis. For each participant, we have determined the mean values and standard deviation of the estimated limit values. To keep confident with the collected data we used both participants' self-report of confidence to find their limits values and the variation of their estimations of these values. Users with high standard deviation (greater than $20 \mathrm{deg}$ ) and who answered that they did not find the limit value were rejected. We considered that such a result and a "no" to the question reflects an indecision. For the 20 participants considered, the mean values are 61.9 deg for the minimum value (standard deviation $12.9 \mathrm{deg}$ ) and $123.2 \mathrm{deg}$ for the maximum value (standard deviation 15.2 $\mathrm{deg}$ ), and the mean value of the standard deviation is $10.8 \mathrm{deg}$ for the minimum value and $17.9 \mathrm{deg}$ for the maximum value.

The users' means of self-estimation of the limit values for visual comfort follow normal laws (see Fig. 3). To verify the assumption of normality for the data's distribution we run the Kolmogorov-Smirnov test for normality, and the Lilliefors test. The lack of significant differences assesses that they are accurately modeled by a normal distribution.

By choosing for each of these normal laws the value at $75 \%$ of probability, we obtain limit values for the minimum (71 deg) and maximum (133 deg) values, which will satisfy $75 \%$ of the population. We suggest accepting these two limits as minimum and maximum values.

The minimum value matches the performances value obtained in test 1 (less than $75 \mathrm{deg}$ ). It is better to use a horizontal FOV higher than 71 deg for the virtual cockpit application, but it is not necessary to go higher than 133 deg. Between these two values, performances will not increase, but comfort will.

Note that test 2 is a subjective test as it is based on user self-assessment. This characteristic is voluntary as it has the

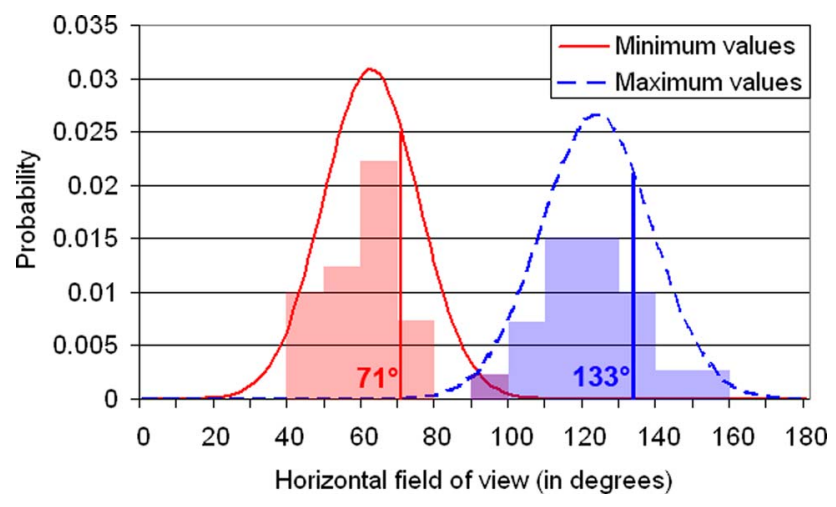

Fig. 3 Normal laws representing the probabilities of the limit values of field of view.

goal of taking into account human visual comfort. That is why standard errors are varied as everyone feels different within the application. However, the two values are well separated and their distribution on normal laws lets us suggest that they exist and are usable.

\section{Conclusion}

We have presented FOV tests in order to determine the necessary and sufficient FOV for a virtual cockpit application, which will be used for ergonomics studies. The results have shown that there are two limit values. The minimum one is $75 \mathrm{deg}$. Under this limit value user performance might be degraded (see test 1), and most of the users find that it is not comfortable enough to perform the tasks of the application for a FOV lower than this value (see test 2). For a FOV higher than $75 \mathrm{deg}$, performance does not increase, but visual comfort does. Most of the users find an enhancement of comfort when FOV increases, for FOV lower than $133 \mathrm{deg}$ (see test 2). It seems that there is no enhancement of comfort for higher FOV.

In conclusion, we advise choosing a value of horizontal FOV higher than $75 \mathrm{deg}$ and as near as possible to $133 \mathrm{deg}$ for a virtual cockpit application for ergonomics studies. Note that these values of FOV are very far from the FOV of good HMD: from 40 deg for good HMD, to 120 deg for lower quality HMD.

Other investigations can be added to this one in the FOV determination: tests on vertical FOV, and on overlap.

In future works, we will also develop interaction for this application, in order to allow the user to interact in an intuitive and natural way with the VMU.

\section{Acknowledgment}

We would like to thank J.M. Burkhardt (from "Laboratoire d'Ergonomie Informatique") for his help on the tests, and P. Philibert and A. Le Hyaric for their help in English.

\section{References}

1. K. W. Arthur, "Effects of field of view on performance with headmounted displays," Thesis Dissertation, University of North Carolina at Chapel Hill, Department of Computer Science (2000).

2. E. F. Kasper, L. A. Haworth, Z. P. Szoboszlay, R. D. King, and Z. L. Halmos, "Effects of inflight view restriction on rotorcraft pilot head movement," Proc. SPIE 3058, 34-45 (1997).

3. J. Schiefele, "Realization and evaluation of virtual cockpit simulation and virtual flight simulation," Thesis Dissertation, University of Technology, Darmstadt (2000). 